This item was submitted to Loughborough's Research Repository by the author.

Items in Figshare are protected by copyright, with all rights reserved, unless otherwise indicated.

\title{
Stochastic unit commitment in microgrids based on model predictive control
}

PLEASE CITE THE PUBLISHED VERSION

http://doi.org/10.1109/SEST.2018.8495736

PUBLISHER

(c) IEEE

VERSION

AM (Accepted Manuscript)

LICENCE

CC BY-NC-ND 4.0

REPOSITORY RECORD

Barrios, Lazaro Alvarado, Juan Boza Valerino, Alvaro Rodriguez del Nozal, Juan Manuel Escano, Jose L. Martinez-Ramos, and Francisco M. Gonzalez-Longatt. 2019. "Stochastic Unit Commitment in Microgrids Based on Model Predictive Control”. figshare. https://hdl.handle.net/2134/34519. 


\section{Stochastic Unit Commitment in Microgrids based on Model Predictive Control}

\author{
Lázaro Alvarado Barrios \\ Dept. of Engineering \\ Universidad Loyola Andalucía \\ Seville, Spain \\ lalvarado@uloyola.es
}

\author{
Juan Manuel Escaño \\ Dept. of Engineering \\ Universidad Loyola Andalucía \\ Seville, Spain \\ jmescano@uloyola.es
}

\author{
Juan Boza Valerino \\ Dept. of Electrical Engineering \\ Universidad de Sevilla \\ Seville, Spain \\ jbozavalerino@gmail.com
}

\author{
José L. Martínez-Ramos \\ Dept. of Electrical Engineering \\ Universidad de Sevilla \\ Seville, Spain \\ camel@us.es
}

\author{
Álvaro Rodríguez del Nozal \\ Dept. of Engineering \\ Universidad Loyola Andalucía \\ Seville, Spain \\ arodriguez@uloyola.es
}

\author{
Francisco Gonzalez-Longatt \\ School of Electronic, Electrical \\ and Systems Engineering \\ Loughborough University \\ Loughborough, United Kingdom \\ fglongatt@fglongatt.org
}

\begin{abstract}
This article deals with the problem of Stochastic Unit Commitment (SUC), considering the stochastic nature of demand and meteorological phenomena. This paper shows the optimal operation of a hybrid microgrid composed of the following generation units: wind turbine (WT), photovoltaic solar panel (PV), diesel engine generator (DE), micro-turbine (MT), as well as storage devices such as Battery Energy Storage (BES), considering its constraints and the requirements of the reserve generation. For this purpose, a Model-based Predictive Control (MPC), which uses dynamic models of prediction of renewable power and demand in real time, is developed, allowing feedback at each step of time, which corrects the uncertainty of the models. A comparison with a classic UC formulation has been made. The results reach a lower cost solution.
\end{abstract}

Index Terms-Stochastic Unit Commitment, Model Predictive Control, Microgrid.

\section{INTRODUCTION}

A microgrid is a bidirectional electric power system that allows the distribution of electricity from suppliers to consumers, favouring the integration of renewable generation sources. It is composed of interconnected loads, conventional (dispatchable) distributed renewable energy generation units and energy storage sources that operate in coordination to supply electricity at the distribution level [1]. It can operate interconnected to the general electrical grid, through one or several Point of Common Coupling (PCC) or it can operate isolated from the grid [2]. A microgrid can generate, store, control and manage part of the energy consumed, allowing the end user not only to be a consumer but also an active part of the grid [3].

Operating in parallel with the main grid, a microgrid can be operated as a single load or aggregate generator and could work as an energy source integrated into the grid or as a way to provide ancillary services that would contribute to the stability and control of the main network [4]. In isolated mode, generators have to be able to respond quickly to changes in consumption so that voltage and frequency remain within acceptable limits. [5].

For microgrids operation planning, the concept of the dispatch strategy is essential. The economic dispatch (ED) contemplates the resolution of the Unit Commiment (UC) initially to determine the schedule with units that must start and stop to respond to the required demand [6]. Once the UC has been proposed, the economic dispatch (ED) is responsible for assigning, to the programmed units, the power references that must be generated to cover the demand at minimum cost at the same time, satisfy the physical constraints of the generating units (power balance, power generation limits) and the grid (power flow and voltage limits) [7].

As a result of the differences between traditional generation systems and microgrids, several publications in recent years have suggested new and different approaches and methods for solving ED and UC problems. For this purpose, for different microgrid structures, metaheuristic and heuristic methods have been proposed and applied to solve the problem of load dispatch [8], [9]. Some advanced control algorithms which consider system uncertainties, caused by demand and renewable sources of energy, have been developed to achieve optimal use of energy storage to compensate for physical imbalances, etc [10], [11], [12], [13]. 
Microgrid operation optimisation is extremely important to manage the energy resources efficiently. Due to the complexity of the optimisation problem and given the economic benefits that are achieved, attention has been paid to the improvement of optimisation algorithms.

Recently, Model-based Predictive Control (MPC) techniques are being applied to power systems. They provide a receding prediction horizon and a feedback mechanism which makes them a more robust tool against uncertainties. They also can handle constraints such as generating capacity and slack power production or consumption, limitation of work cycles and battery charging limits, etc.

In this article, the UC problem, considering the stochastic nature of the demand and the weather phenomena, is tackled. A novel type of electric market is considered within the microgrid in which the required demand is met with the power generated by the available local generation resources in real time. For this purpose, a Model Predictive Control (MPC) is solved at every time step to cover the demand regarding the uncertainties introduced by the prediction in the power variables. The hybrid microgrid is composed of generation units: wind turbine (WT), solar photo-voltaic panel (PV), diesel engine generator (DE), micro-turbine (MT), as well as storage devices such as Battery Energy Storage (BES), considering their constraints and the requirements of the generation reserve. The cost coefficients and constraints of conventional dispatchable units, renewable energy sources and BES are taken as data to solve the problem of stochastic unit commitment (SUC). The article is organised as following, section II presents the MPC formulation, presenting the cost function and the constraints. In section IV a case study will be used to compare the results obtained by a conventional scheduling based on forecasts performed only at the start of the scheduling period, and the proposed MPC strategy. The conclusion is given in section $\mathrm{V}$.

\section{MPC STRATEGY}

In MPC, the controller runs a real-time optimisation to obtain the best solution for the control action [14]. MPC uses a dynamic model to predict the future behavior of the system. Setting a period of time of prediction (prediction horizon), an optimisation problem is solved to identify the best control action that minimizes a cost function related to the process predictions (see ofr instance Figure 1). In this case, the prediction model includes the selection of the generating units including the BES. The committment status of the dispatchable resources is determined by the value of the binary variable $\delta_{i t} \in\{0,1\}$ where subindex $i$ denotes the resource and subindex $t$ denotes the time instant. For this study and regarding the low power capability of the generation units we will consider a negligible startup and shutdown time. Since all the generating units are dispatchable, the value 1 indicates commitment and 0 indicates shut down. In the case of the BES that has three working modes, 1 indicates charging or discharging and the value 0 indicates that the BES is in standby (pause). The idea behind such control strategy is the optimisation of an objective function, calculating the appropriate sequence of inputs over a prediction horizon based on a model of the plant. Only the first control set point $u(1)$ is sent to the device and the whole process is repeated for the next sampling instant. Thus, continuous feedback of the control variable is obtained. The prediction horizon is always the same in each sample (a common thing in predictive control strategies). The difference is, with respect to any other optimal control strategy, the MPC is a receding horizon optimal control.

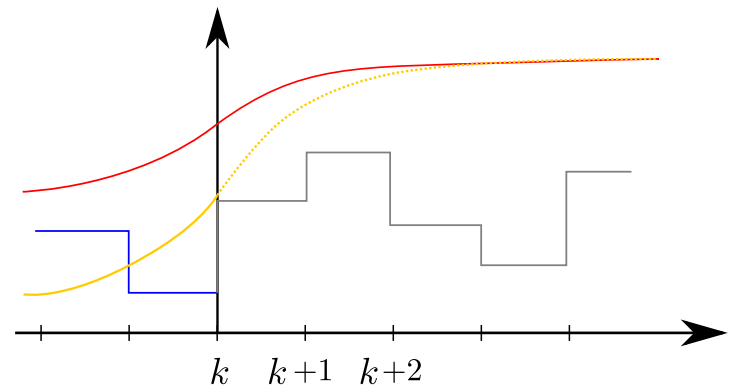

Fig. 1. Based on a model of the plant, the MPC algorithm solves an optimization problem to choose the best input to the system according to certain parameters. The figure shows a desired trajectory for a plant (red line). The blue and green lines represent the past input to the system and the past plant state, respectively. With that information the MPC solves the problem choosing a set of future control inputs (gray) and predicting the future state of the plant (green with dashed lines)

This continuous feedback allows errors to be assumed in the models. In fact, the stochastic nature of renewable energy production and demand makes MPC an adequate strategy against static planning through optimization. In the case of the microgrid, due to the lower granularity concerning the zone and the behavior of the demand, the uncertainty in the prediction is a growth factor with the prediction horizon. Figure 2 shows a set of real demand data, wind and solar power. The predictions have been artificially generated by adding an increasing uncertainty over time to the real data, to provoke a difference between predicted and real values when there are prediction uncertainties, as shown in graphs (b) and (c) in weather forecasts and the increasing error that the ARMA model used to predict demand, as shown in the graph (a).

\section{SYSTEM MODELING}

In this section the model of our problem is presented. We consider a hybrid microgrid composed of four generation units: wind turbine (WT), solar photo-voltaic panels (PV), diesel engine generator (DE) and a micro-turbine (MT). A battery energy storage (BES) system is also connected to the microgrid allowing us to use it as a generator or as a load in order to minimize the total cost of operation. 

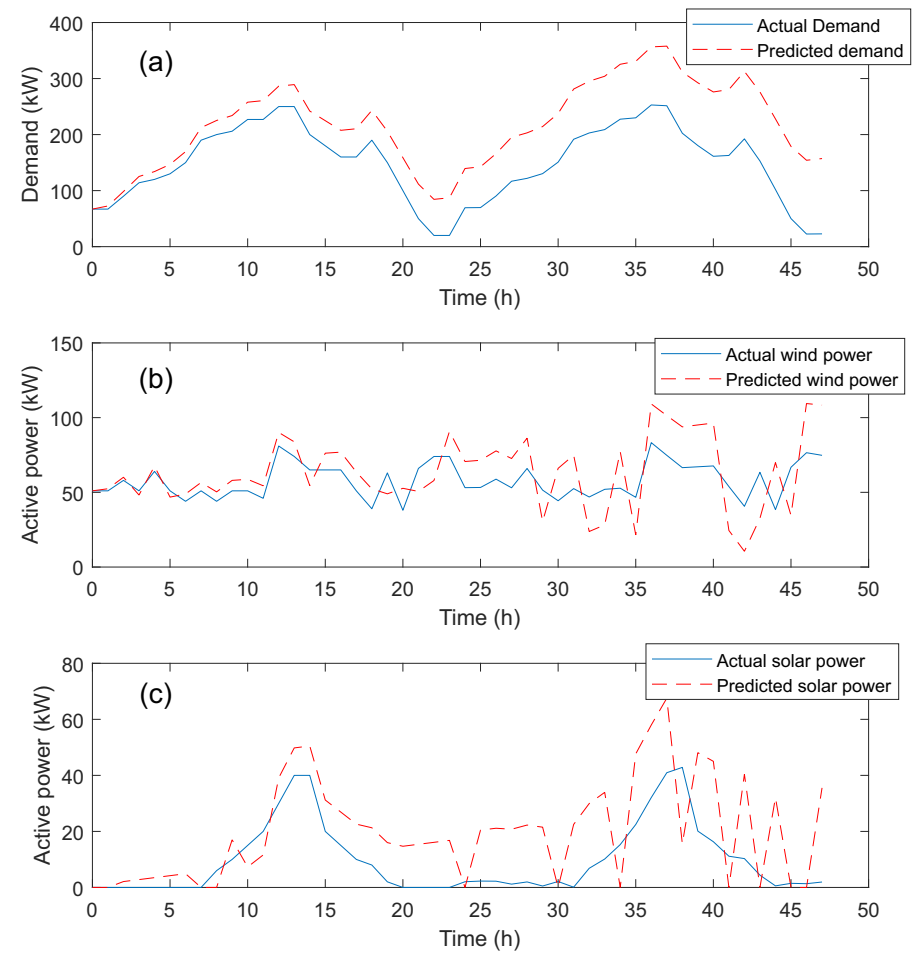

Fig. 2. Prediction models of renewable power and demand

\section{A. Objective function}

The main aim of our algorithm is to reduce the total cost of operation of the system. To do that let us consider the following cost function:

$$
\min \sum_{i=D E, M T}\left(\sum_{t=k}^{k+N-1}\left(a_{i} \delta_{i t}+b_{i} P_{i t}\right)\right)
$$

where $P_{i t} \in \mathbb{R}$ is the power generated by the resource $i$ at time instant $t ; N$ is the prediction horizon of the MPC; and $a_{i}$ and $b_{i}$ are the startup and the marginal cost of generation unit $i$, respectively.

\section{B. Constraints}

The problem is subject to some physical contraints that must be fulfilled.

- Power balance

The total generation of all dispatchable distributed generators (DE,MT), renewable generation units (WT, PV) and BES must be equal to the system demands at every time step considered.

$$
P_{t}^{d}=P_{D E, t}+P_{M T, t}+P_{W T, t}+P_{P V, t}+P_{B E S, t}
$$

for all $t \in[k, k+N-1]$.

- Generation limits

The generation of each unit is bounded according to physical constraints:

$$
\underline{P}_{i, t} \delta_{i, t} \leq P_{i, t} \leq \bar{P}_{i, t} \delta_{i, t} \quad \forall i,
$$

Where $\underline{P}_{i, t}$ and $\bar{P}_{i, t}$ are the maximum and minimum amount of power that can be generated by generator $i$, respectively.

- Storage constraints

We define $S O C_{t}$ as the state of charge of the battery at time $k$. The state of the charge of the battery is bounded according to the following inequality:

$$
\underline{S O C} \leq S O C_{t} \leq \overline{S O C} \quad \forall t \in[k, k+N-1] .
$$

Additionally, the state of the battery has the following dynamics:

$$
S O C_{t}=S O C_{t-1}-P_{B E S, t} \Delta t
$$

where $\Delta t$ is the time between to consecutive time steps. Please, note that for this case of study we are considering an ideal battery storage system in which the charging and discharging efficiency is $100 \%$.

Finally, it is worth pointing out that the generation of the battery at one concrete time instant is limited according to $(3)$.

\section{Simulation and Results}

This section presents the simulations results of the problem considered. The structure of the simulated microgrid is shown in Fig. 3. As it was mentioned before, it consists of a diesel engine (DE), a wind turbine (WT), a photovoltaic panel (PV), a diesel engine generator (DE) and a BES. The cost of fuel consumed by each disposable unit is modeled by a linear function. The Tables I and II show the technical and economic characteristics of each asset that forms the microgrid.

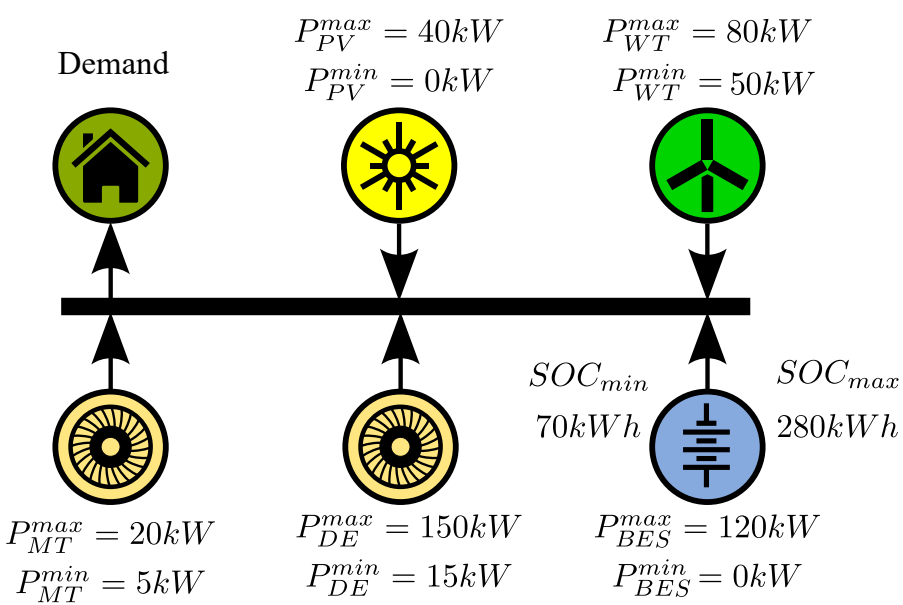

Fig. 3. Structure of the microgrid of the case study

\section{A. Simulations results}

In this case, for the MPC, prediction models such as those shown in Figure 2 have been used, where prediction errors increase over time. Figure 4 shows a comparison between a conventional UC, made by using the prediction models obtained at the first moment, and dynamic planning, based 
TABLE I

TECHNICAL DATA OF GENERATOR UNITS [11]

\begin{tabular}{ccccc}
\hline Sources & $\begin{array}{c}\mathrm{P}_{\min } \\
(\mathrm{kW})\end{array}$ & $\begin{array}{c}\mathrm{P}_{\max } \\
(\mathrm{kW})\end{array}$ & $\begin{array}{c}\mathrm{SOC}_{\min } \\
(\mathrm{kWh})\end{array}$ & $\begin{array}{c}\mathrm{SOC}_{\max } \\
(\mathrm{kWh})\end{array}$ \\
\hline $\mathrm{DE}$ & 15 & 150 & & \\
\hline $\mathrm{PW}$ & 50 & 80 & & \\
\hline $\mathrm{PV}$ & 0 & 40 & & 280 \\
\hline $\mathrm{BB}$ & 0 & 120 & 70 & \\
\hline
\end{tabular}

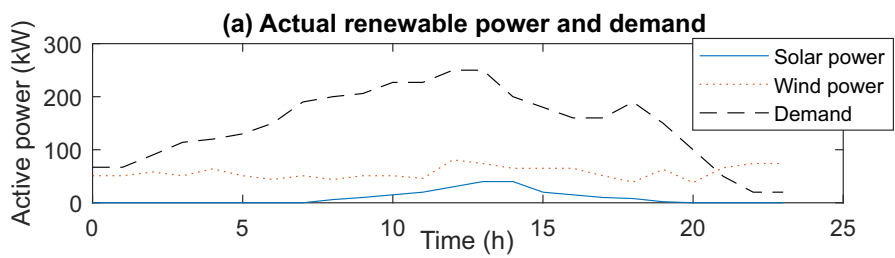

TABLE II

COSTS COEFFICIENTS OF GENERATOR UNITS [11]

\begin{tabular}{ccc}
\hline \multirow{2}{*}{ DDG } & \multicolumn{2}{c}{ Coefficient Cost } \\
\cline { 2 - 3 } & $\begin{array}{c}\mathrm{a} \\
(€)\end{array}$ & $\begin{array}{c}\mathrm{b} \\
(€ / k W h)\end{array}$ \\
\hline $\mathrm{DE}$ & 0,6 & 0,05 \\
\hline
\end{tabular}

on MPC, where at each sampling time (1 hour), Optimization is recalculated with new prediction models, displacing the time horizon ahead. Several issues can be observed:

- We can see in Figure 4(b) that MT is kept disconnected in both strategies, since its cost is higher than the other assets and the demand can be covered by them.

- In Figure 4(c), DE is operated less time and with less power by MPC strategy; it will result at less cost.

- Figure 4(d) shows that MPC strategy keeps the battery at more stable operation, since the demand is higher than the renewable production and the MPC searches for the optimum in each sample time, expending as much as possible from the battery. Nevertheless, this operational mode will increase the life of the BES device.

- We can observe in 4(e) how the MPC strategy keeps the SOC of the battery bank in optimum levels, avoiding full charges-discharges cycles, like in the Non-MPC strategy

- The simple UC strategy connects longer time the DG, being more expensive than the MPC scheduling. Figure 5 shows the contribution of dispatchable, renewable and energy storage systems.

- Finally, it can be seen in Table IV-A the final value of cost function (1) for conventional battery management strategies and using MPC. It can be seen that the MPC improves the performance of the problem achieving a better solution.

In the Figures 6 (a) and (b), the different power states of the battery bank and diesel group (in the rows from bottom to top) are shown according to the sampling time (columns). Thus, it can be seen in Figure 6 (a), how the evolution of the battery management in the prediction horizon evolves, for instance, if we take column one, we can see the predicted power used by the battery in the following 24 hours, but only the action at time instant $k$ is considered. Next, if we take the sequel column, we have the predictions of power from hour 1 to hour 25. It is worth mentioning that these predictions are not the same than the ones estimated at the previous instant due to the perturbations considered. The same reasoning works also for 6 (b), where the predictions of the power generated with the DE are shown.
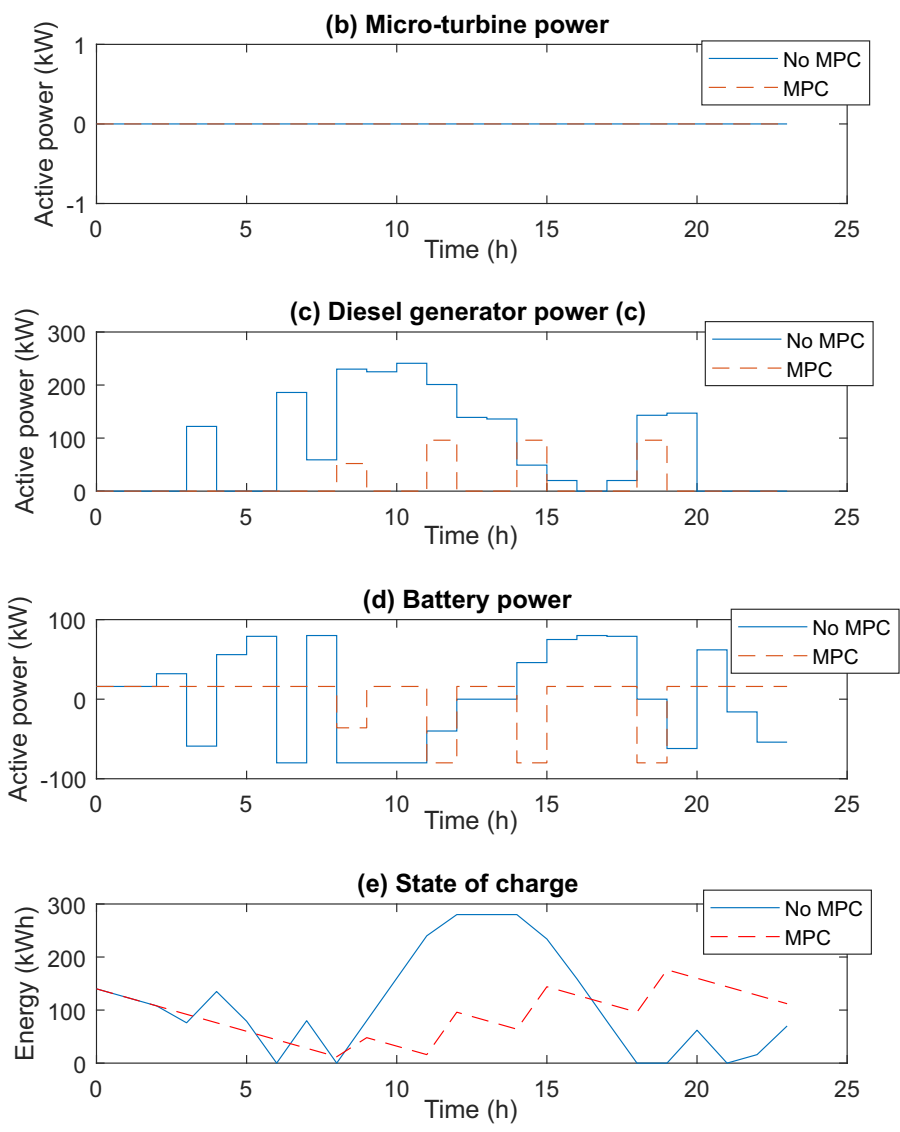

Fig. 4. Simulation results

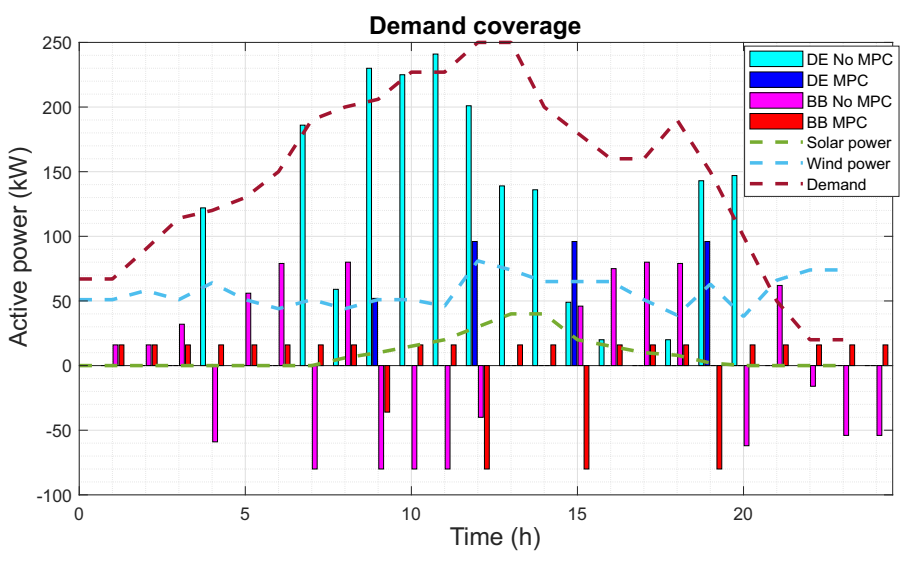

Fig. 5. Demand coverage by the available resources. 

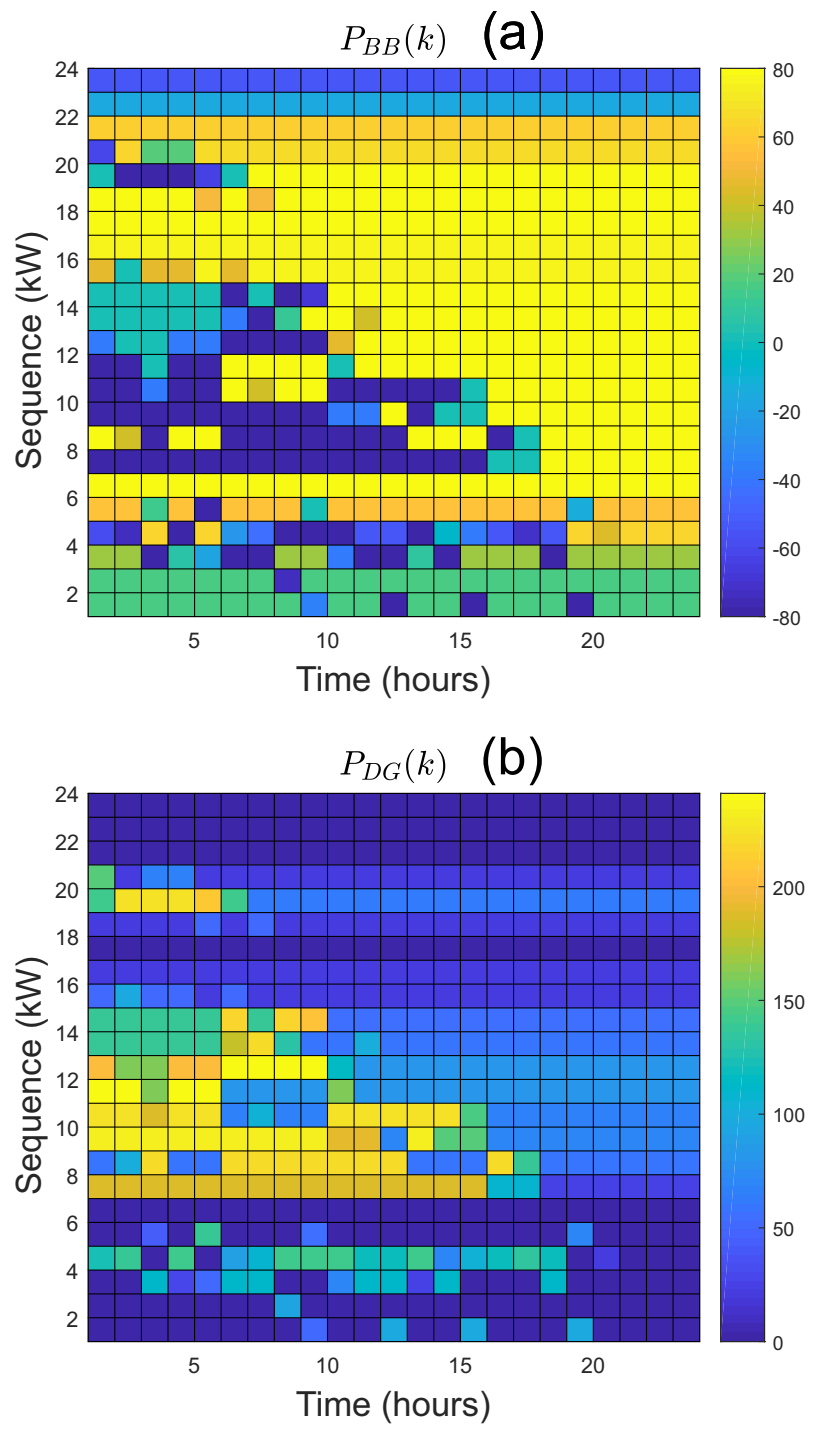

Fig. 6. Batery bank (a) and Diesel generator (b) power sequences

TABLE III

TOTAL COSTS/DAY (€) PER CONTROL STRATEGY

\begin{tabular}{cc}
\hline Without MPC & $€ 104.3$ \\
\hline With MPC & $€ 19.4$ \\
\hline
\end{tabular}

\section{CONCLusion ANd Future Work}

An MPC strategy has been tested in a microgrid to obtain a minimum operation cost, compared to traditional UC, with a single optimization problem. The MPC has shown a better performance against modeling errors present in the predictions of demand and weather forecast.

A further work it will be interesting to explore the use of robust predictive control, taking into account in the optimization, the uncertainties of the problem guaranteeing stability. Additionally, the consideration of the model of the electrical grid will be considered also as a future work.

\section{ACKNOWLEDGEMENT}

The authors would like to acknowledge the financial support of the Spanish Ministry of Economy and Competitiveness under Grants PCIN-2015-043 and ENE2015-69597-R and AEI/FEDER by grant TEC2016-80242-P.

\section{REFERENCES}

[1] Hatziargyriou, Nikos D. and Kleftakis, Vasilis A. and Papadimitriou, Christina N. and Messinis, George M., "Microgrids in Distribution", Smart Grid Handbook, Wiley Online Library, pp. 1-24, 2016.

[2] Parhizi, Sina and Lotfi, Hossein and Khodaei, Amin and Bahramirad, Shay, "State of the art in research on microgrids: A review", Ieee Access, vol. 3, pp. 890-925, 2015.

[3] Parisio, Alessandra and Rikos, Evangelos and Tzamalis, George and Glielmo, Luigi, "Use of model predictive control for experimental microgrid optimization”, Applied Energy, vol. 115, pp. 37-46, 2014.

[4] Shah, Jalpa and Wollenberg, Bruce F and Mohan, Ned, "Decentralized power flow control for a smart micro-grid", Power and Energy Society General Meeting, 2011 IEEE, pp. 1-6, 2011.

[5] Olivares, Daniel E and Cañizares, Claudio A and Kazerani, Mehrdad, "A centralized energy management system for isolated microgrids", IEEE Transactions on smart grid, vol. 5, n 4, pp. 1864-1875, 2014.

[6] Zheng, Qipeng P and Wang, Jianhui and Liu, Andrew L, "Stochastic optimization for unit commitmentA review", IEEE Transactions on Power Systems, vol. 30, n 4, pp. 1913-1924, 2015.

[7] Dai, Hang and Zhang, Ni and Su, Wencong, "A literature review of stochastic programming and unit commitment", Journal of Power and Energy Engineering, vol.3, n 4, pp. 206-214, 2015.

[8] Nguyen, Minh Y and Yoon, Yong T and Choi, Nack H, "Dynamic programming formulation of micro-grid operation with heat and electricity constraints", Transmission \& Distribution Conference \& Exposition: Asia and Pacific, 2009, IEEE, pp. 1-4, 2009.

[9] Liang, HZ and Gooi, HB, "Unit commitment in microgrids by improved genetic algorithm", IPEC, 2010 Conference Proceedings, IEE, pp. 842847, 2010.

[10] Talari, Saber and Yazdaninejad, Mohsen and Haghifam, Mahmoud-Reza, "Stochastic-based scheduling of the microgrid operation including wind turbines, photovoltaic cells, energy storages and responsive loads", IET Generation, Transmission \& Distribution, vol. 9, n12, pp. 1498-1509, 2015.

[11] Raj, Vinod M and Chanana, Saurabh, "Fuel cost optimization of an islanded microgrid considering environmental impact", Journal of Clean Energy Technologies, vol. 4 n 2, pp. 120-124, 2016.

[12] el Motaleb, Ahmad Mohamed Abd and Bekdache, Sarah Kazim and Barrios, Lázaro Alvarado, "Optimal sizing for a hybrid power system with wind/energy storage based in stochastic environment", Renewable and Sustainable Energy Reviews, vol. 59, pp. 1149-1158, 2016.

[13] Deckmyn, Christof and Van de Vyver, Jan and Vandoorn, Tine L and Meersman, Bart and Desmet, Jan and Vandevelde, Lieven, "Day-ahead unit commitment model for microgrids", IET Generation, Transmission \& Distribution, vol.11, n 1, pp. 1-9, 2017.

[14] Bordons C., Camacho, E.F. "Model Predictive Control. Second Edition. Ed. 2. Londres,. Springer-Verlag. 2004. 426. ISBN 1-85233-694-3 